ARTIGO ORIGINAL

ORIGINAL ARTICLE

\section{Análise do impacto econômico da falta de suplementação multivitamínica em mulheres em idade reprodutiva e suas consequências em recém-nascidos}

\author{
Analysis of the economic impact of lack of vitamin \\ supplementation on women at childbearing age \\ and the repercussions on the newborns
}

Mariana Del Aguila' ${ }^{1}$ Adriano Bueno Tavares², Roberta Arinelli³ Ana Carolina Padula Ribeiro Pereira ${ }^{3}$, Camila Pepe ${ }^{3}$

DOI: $10.21115 / J B E S . v 12 . n 2 . p 135-41$

\section{Palavras-chave:}

micronutrientes, suplementos nutricionais, gravidez, recém-nascido, custos e análise de custo

\section{Keywords:}

micronutrients, dietary

supplement, pregnancy, newborn, cost and cost analysis

\begin{abstract}
RESUMO
Objetivo: Avaliar o impacto econômico da falta de suplementação multivitamínica de mulheres no período reprodutivo e suas consequências em recém-nascidos, por meio da estimativa do número de casos e dos custos implicados no tratamento de complicações decorrentes da falta de suplementação, sob a perspectiva do sistema público de saúde. Métodos: A população foi definida a partir do número de gestantes e nascidos vivos no Brasil, ajustado para o ano de 2019, e de pessoas que utilizam exclusivamente o SUS, considerando um crescimento linear. Por meio de revisão de literatura, os riscos de desenvolvimento de defeitos do fechamento do tubo neural, pré-eclâmpsia e anemia foram determinados. Para a estimativa dos custos diretos médicos, realizou-se um microcusteio com base na utilização de recursos determinados por condutas médicas estabelecidas e opiniões de especialistas. A ocorrência de eventos e os respectivos custos foram calculados multiplicando o número de indivíduos pelo risco de desenvolvimento das complicações e os custos individuais pela quantidade de eventos. Resultados: $O$ custo total da falta de suplementação vitamínica foi estimado em 2,47 bilhões de reais. A falta de suplementação vitamínica seria capaz de gerar a ocorrência anual de 5.352, 796.283, 298.801 e até 345.628 casos de defeitos do fechamento do tubo neural, anemia, parto prematuro e pré-eclâmpsia, respectivamente. Conclusões: A ausência de suplementação vitamínica pode gerar um custo anual de até 2,47 bilhões de reais para o sistema público de saúde brasileiro, com ocorrência de pelo menos 796 mil casos de complicações decorrentes por ano.
\end{abstract}


risk of developing complications and individual costs by the number of events. Results: Total cost of vitamin supplementation absence in this group was estimated at 2.47 billion Brazilian real (BRL). The absence of vitamin supplementation would be able to generate annually 5,352, 796,283, 298,801, and up to 345,628 cases of neural tube closure defects, anemia, preterm delivery and preeclampsia, respectively. Conclusions: The lack of vitamin supplementation can generate an annual cost of 2,47 billion BRL for the Brazilian public health system with at least 796,000 cases of complications arising per year.

\section{Introdução}

A deficiência de micronutrientes é um problema no âmbito da saúde pública e, de acordo com a Organização Mundial da Saúde (OMS), afeta cerca de 2 bilhões de pessoas ao redor do mundo (Unicef, 2009). Os efeitos decorrentes dessa deficiência em gestantes afetam principalmente os recém-nascidos, podendo resultar em morte prematura, saúde precária, deficiência visual, além de efeitos negativos a longo prazo, como atraso no crescimento e desenvolvimento cognitivo, dificuldades de aprendizado e baixa capacidade laboral (Howson et al., 1998). Nesse contexto, a importância da ingestão de micronutrientes pela mulher nos períodos pré-concepcional, gestacional e durante a amamentação tem sido levantada por diferentes autores (Karakochuk et al., 2018; Mozetic et al., 2016).

O período gestacional representa uma fase relacionada a diversos ajustes fisiológicos e anatômicos, para que sejam garantidos o crescimento e o desenvolvimento saudável do feto, o que gera uma alteração nas reservas biológicas do organismo materno (Mozetic et al., 2016; Soma-Pillay et al., 2016). Para alguns autores, além dos 1.000 dias que compreendem a gestação e o período de amamentação, o período pré-gestacional representa uma importante janela de oportunidades para a suplementação de micronutrientes, sendo capaz de prevenir defeitos congênitos e futuros problemas na saúde da criança (Czeizel, 1998; Hanson et al., 2015).

A suplementação de micronutrientes por diferentes estratégias, tais como ácido fólico, ferro, zinco, magnésio, vitaminas D e E, cálcio e ômega-3, tem sido amplamente discutida na literatura. Estudos apresentam resultados de eficácia e segurança satisfatórios, tanto para desfechos relacionados às mulheres, como pré-eclâmpsia e anemia materna, quanto para aqueles relacionados às crianças, como defeitos de fechamento do tubo neural e prematuridade (Czeizel, 1998; De-Regil et al., 2016; Hofmeyr et al., 2018; Makrides et al., 2014; Middleton et al., 2018; Ota et al., 2015; Peña-Rosas et al., 2015; Rumbold et al., 2015a; Rumbold et al., 2015b; Salam et al., 2015).

A relação entre os defeitos do fechamento do tubo neural e a suplementação com ácido fólico antes da concepção e durante o primeiro trimestre de gestação é amplamente abordada na comunidade científica. Os defeitos do fechamento do tubo neural são malformações congênitas que ocorrem devido a uma falha no fechamento adequado do tubo neural embrionário, durante a quarta semana de embriogênese ou a sexta semana de gestacional, podendo acarretar abortamento, anencefalia e espinha bífida (Aguiar et al., 2003). É estimado que na América Latina a ocorrência desses defeitos varie de 0,2 a 9,6 a cada 1.000 nascidos vivos e que a fortificação de alimentos com ácido fólico seja capaz de reduzir os casos em até 59\% (Rosenthal et al., 2014). Para o Brasil, as estimativas variam de acordo com os estados, de 0,55 a 9,6 a cada 1.000 nascidos vivos (Rosenthal et al., 2014; Santos et al., 2016). Apesar dos esforços de suplementação de ácido fólico por meio da alimentação, especialmente pela fortificação de alimentos, as repercussões decorrentes do defeito no fechamento do tubo neural são capazes de gerar importante impacto social e econômico em diferentes países ao redor do mundo (Yi et al., 2011).

Entre as principais complicações em gestantes que poderiam ser prevenidas pela suplementação vitamínica com cálcio e vitaminas $D, C$ e E, estão os distúrbios hipertensivos, que são a principal causa de mortalidade materna na América Latina, inclusive no Brasil (Say et al., 2014). A pré-eclâmpsia, o principal distúrbio hipertensivo em gestantes, é caracterizada por uma resposta vascular anormal à presença da placenta associada a um aumento da resistência vascular sistêmica, agregação plaquetária exacerbada, ativação do sistema de coagulação e disfunção das células endoteliais (Sibai et al., 2005). A estimativa de ocorrência de pré-eclâmpsia, em gestantes em geral, varia de 2 a 8 casos a cada 1.000 nascidos vivos, e essa característica está associada à maior ocorrência de trombocitopenia, descolamento de placenta, coagulação intravascular disseminada e pneumonia, entre outras complicações maternas e fetais (Sibai et al., 2005). Estudos demonstram que o uso de suplementação multivitamínica, principalmente com vitamina $D$, durante a gestação pode reduzir o risco de ocorrência de pré-eclâmpsia, independentemente do tipo (Fu et al., 2018). No Brasil, a taxa de mortalidade materna por eclâmpsia, forma mais grave da pré-eclâmpsia, é estimada em 19,5 por 100.000 nascidos vivos, sendo maior em regiões com menor Índice de Desenvolvimento Humano (Giordano et al., 2014).

Além dos distúrbios hipertensivos, a anemia por deficiência de ferro configura uma importante carência nutricional, sendo as gestantes um dos grupos mais suscetíveis, devido à elevada necessidade de ferro, determinada pela rápida expansão dos tecidos e da produção de hemácias (Fujimori et al., 2011). A prevalência de anemia no Brasil é estimada em $29,4 \%$ entre mulheres em idade fértil e 20,9\% em crianças com menos de 6 anos de idade. Ela geralmente está associada à deficiência de ferro, é estimada em 29,4\% e 20,9\%, 
respectivamente, e varia de 4\% a 61,3\% em países da América Latina (Mujica-Coopman et al., 2015).

Além da deficiência de ácido fólico e ferro, a carência de outras vitaminas pode gerar importantes impactos para as mulheres em idade fértil e seus conceptos, no entanto dados de prevalência e custos associados ao seu manejo no Brasil ainda são pouco conhecidos.

No Brasil, cerca de 3 milhões de nascimentos ocorrem por ano, considerando apenas nascidos vivos (Brasil, n.d.). Sendo assim, este estudo foi conduzido com o objetivo de avaliar o impacto da falta de suplementação de micronutrientes, por meio da estimativa do número de casos potenciais da deficiência de um micronutriente e dos custos implicados no tratamento de complicações em mulheres em idade fértil e recém-nascidos afetados, sob a perspectiva do sistema público de saúde brasileiro.

\section{Materiais e métodos}

\section{População de interesse}

Para esta análise, foi considerado o número médio de gestantes e de nascidos vivos no Brasil, estimado de acordo com cálculos realizados a partir de dados sobre o número de nascimentos por mãe, obtidos nas estatísticas de 2010 a 2016, divulgadas pelo Governo Federal (Brasil, n.d.).

\section{Frequência de complicações e deficiência de vitaminas}

Para estimar o efeito da suplementação no período de interesse, os riscos de desenvolvimento de defeitos do fechamento do tubo neural (espinha bífida, anencefalia e interrupção da gestação), pré-eclâmpsia, prematuridade e anemia foram extraídos de dados previamente publicados. Para isso, foi inicialmente conduzida uma revisão de literatura na base de dados da biblioteca Cochrane até maio de 2019, com o objetivo de localizar revisões sistemáticas e/ou metanálises acerca da eficácia de diferentes tipos de suplementação vitamínica para os diversos desfechos de interesse. Caso não fossem localizados estudos com os desfechos de interesse, seriam consultadas as bases de dados Medline via PubMed e Literatura Latino-Americana e do Caribe em Ciências da Saúde (Lilacs).

\section{Utilização de recursos e estimativas de custo}

Os custos diretos médicos foram estimados por meio de microcusteio, com base em condutas estabelecidas para a determinação da utilização de recursos. Dessa forma, os custos anuais relacionados ao manejo das complicações, sob a perspectiva do Sistema Único de Saúde (SUS), foram estimados.

Os custos das complicações foram calculados como a soma dos custos relacionados ao seu manejo, considerando cada item a ser utilizado. Esses itens foram determinados de acordo com a opinião de especialistas na área. Para a precificação, fontes oficiais de preço foram consultadas: Sistema de Gerenciamento da Tabela de Procedimentos, Medicamentos e OPM do SUS (SIGTAP), programa TabNet e Sistema de Informações Ambulatoriais do SUS (SIASUS). Apenas para o manejo da prematuridade, os custos foram extraídos do estudo publicado por Desgualdo e colaboradores (2011) (Desgualdo et al., 2011). Os custos foram expressos em real brasileiro para o ano de 2019.

\section{Perspectiva}

Foi adotada a perspectiva do sistema público de saúde brasileiro (SUS) como fonte pagadora de serviços de saúde.

\section{Estrutura e características do modelo}

A estimativa de ocorrência de eventos pela falta de suplementação vitamínica foi calculada multiplicando o número de gestantes e nascidos vivos pelo risco de desenvolvimento de defeitos do fechamento do tubo neural, pré-eclâmpsia, prematuridade e anemia.

A partir dos dados de probabilidade obtidos na revisão sistemática, foi possível estimar a quantidade de eventos ocorridos para cada desfecho de interesse, de acordo com o uso ou não de suplementação. A falta de suplementação com ácido fólico foi relacionada à ocorrência de defeitos do fechamento do tubo neural, à falta de suplementação com ferro, com a ocorrência de anemia materna (hemoglobina $<$ 11,0 g/dL em 37 semanas ou mais), e à falta de suplementação com ômega-3, com a ocorrência de parto prematuro (<37 semanas). Já a ocorrência de pré-eclâmpsia foi relacionada à falta de suplementação com cálcio, magnésio, zinco e vitaminas $C$, D e E.

A estimativa de custos com esses eventos foi calculada a partir da quantidade estimada de cada evento pelo custo individual de cada evento.

\section{Análise estatística}

A análise dos dados obtidos no estudo foi realizada de maneira descritiva, utilizando valores médios e percentuais, dependendo do tipo de variável apresentada. Para essas análises, o programa Microsoft Excel 2016 foi utilizado.

\section{Resultados}

Para a estimativa da população SUS de gestantes e de nascidos vivos no ano de 2019, realizou-se um ajuste considerando um crescimento linear com base no histórico de gestantes e nascidos vivos na base do Sinasc (Sistema de Informação sobre Nascidos Vivos) ao longo do período de 2010 a 2016, último dado disponível na base. As equações que definem o número de gestantes e de nascidos vivos para 2019 foram calculadas em Microsoft Excel e estão apresentadas a seguir.

Equação 1. Equação linear para definir número de gestantes e nascidos vivos

Gestantes $->Y=9273 X+2.852 .919$

Nascidos vivos $->Y=9653 X+2.881 .327$ 
Onde Y é o número de gestantes/nascidos vivos e X é o número inteiro que representa o período de tempo que se deseja analisar (por exemplo, 2010 equivale ao número inteiro 1, 2011 ao 2, 2012 ao 3 e 2019 ao 10). A população total de gestantes e nascidos vivos foi reduzida da taxa de cobertura do sistema privado de saúde, de 24,3\% (ANS, 2019), considerando-se que na vida real gestantes atendidas pelo sistema privado não utilizariam o sistema público de saúde brasileiro para tratamentos e acompanhamentos gestacionais, pós-gestacionais e pediátricos para seus filhos.

Assim, a população total de gestantes estimada para 2019 no sistema público de saúde é de 2.229 .860 e de nascidos vivos, de 2.254.237.

\section{Desfechos}

A Tabela 1 apresenta a probabilidade de ocorrência dos desfechos considerados na análise. Os desfechos foram observados em gestantes e recém-nascidos que não realizaram suplementação de micronutrientes; os dados foram extraídos de estudos selecionados na revisão de literatura.

A Tabela 2 apresenta os dados de qualidade de vida utilizados para a construção do modelo para indivíduos com espinha bífida, de acordo com a altura da lesão. A lesão na altura da coluna lombar é a mais frequentemente observada (62\%). Apesar da população apresentar expectativa de vida similar (72 anos para pacientes com lesão lombar e 79 anos para pacientes com lesão sacral), a quantidade de anos de vida ajustados pela qualidade (QALY - Quality-Adjusted Life Years) estimados é aproximadamente a metade daqueles apresentados pelos indivíduos com lesão sacral (30,12 para pacientes com lesão lombar e 61,42 para pacientes com lesão sacral). Para pacientes com espinha bífida, independentemente da altura da lesão, foi estimado um total de 27,76 QALY, resultante da média dos QALY para cada tipo de lesão ponderada pela proporção de pacientes com cada tipo de lesão.

Os custos médios do manejo de cada um dos eventos por pessoa, considerados para o cálculo do custo total atribuído à falta de suplementação, estão descritos na Tabela 3. Os valores variaram de R\$17.949,95, para o manejo de defeitos do fechamento do tubo neural, a R\$155,22, para o manejo da anemia.

A Tabela 4 e a Tabela 5 apresentam os resultados acerca da estimativa de ocorrência de eventos e os custos com o manejo de complicações associadas à falta da suplementação vitamínica em mulheres no período reprodutivo e recém-nascidos, considerando desfechos como defeitos do fechamento do tubo neural, pré-eclâmpsia, anemia e parto prematuro. O custo total da falta de suplementação vitamínica nesse grupo foi estimado em $\mathrm{R} \$ 2.469 .480 .853$, considerando apenas o custo da deficiência de vitamina D para a ocorrência de pré-eclâmpsia, a fim de não sobrepor custos com uma mesma complicação.

Tratando-se de defeitos do fechamento do tubo neural, a falta de suplementação com multivitamínicos que apresentem ácido fólico em suas formulações resultaria na ocorrência de 5.352 casos por ano, gerando um custo de 96,062 milhões de reais para o sistema público. Os defeitos do fechamento do tubo neural apresentam um espectro clínico variável, sendo os mais comuns a anencefalia e a espinha bífida. Considerando que todas as crianças anencéfalas são natimortas ou morrem pouco tempo após o nascimento (Aguiar et al., 2003), a análise considerou apenas custos com o parto dessas crianças. Para crianças nascidas com espinha bífida, foram considerados os gastos por toda a vida, visto que esse é um desfecho com consequências para a vida inteira.

Tabela 1. Probabilidade de ocorrência dos desfechos de interesse na ausência de suplementação vitamínica, conforme revisão da literatura

\begin{tabular}{|c|c|c|c|c|}
\hline \multirow[b]{3}{*}{ Referência } & \multirow[b]{3}{*}{ Desfecho } & \multirow[b]{3}{*}{$\begin{array}{l}\text { Micronutriente } \\
\text { analisado }\end{array}$} & \multirow{2}{*}{$\begin{array}{c}\text { Gestantes } \\
\text { Status da } \\
\text { suplementação }\end{array}$} & \multirow{2}{*}{$\begin{array}{c}\begin{array}{c}\text { Nascidos vivos } \\
(0-6 \text { meses })\end{array} \\
\begin{array}{c}\text { Status da } \\
\text { suplementação }\end{array}\end{array}$} \\
\hline & & & & \\
\hline & & & $\begin{array}{c}\text { Ausente } \\
\text { (\%) }\end{array}$ & $\begin{array}{l}\text { Ausente } \\
\text { (\%) }\end{array}$ \\
\hline YHEC, 2015 & Defeito de fechamento do tubo neural & Ácido fólico & 0,24 & - \\
\hline Hofmeyr et al., 2018 & Pré-eclâmpsia & Cálcio & 6,5 & - \\
\hline Makrides et al., 2014 & Pré-eclâmpsia & Magnésio & 7,9 & - \\
\hline Rumbold et al., 2015a & Pré-eclâmpsia & Vitamina C & 9,3 & - \\
\hline De-Regil et al., 2016 & Pré-eclâmpsia & Vitamina D & 15,5 & - \\
\hline Rumbold et al., 2015b & Pré-eclâmpsia & Vitamina $\mathrm{E}$ & 9,5 & - \\
\hline Ota et al., 2015 & Pré-eclâmpsia & Zinco & 7,1 & - \\
\hline Peña-Rosa et al., 2015 & $\begin{array}{l}\text { Anemia materna (hemoglobina } \\
<110 \mathrm{~g} / \mathrm{L} \text { em } 37 \text { semanas ou mais) }\end{array}$ & Ferro & 35,7 & - \\
\hline Middleton et al., 2018 & Parto prematuro (<37 semanas) & Ômega-3 & 13,4 & - \\
\hline
\end{tabular}


Tabela 2. Dados de qualidade de vida utilizados para a construção do modelo para indivíduos com espinha bífida de acordo com a altura da lesão

\begin{tabular}{lccc}
\hline & $\begin{array}{c}\text { Lesão } \\
\text { torácica }\end{array}$ & $\begin{array}{c}\text { Lesão } \\
\text { lombar }\end{array}$ & $\begin{array}{c}\text { Lesão } \\
\text { sacral }\end{array}$ \\
\hline Proporção* $^{*}$ & $28 \%$ & $62 \%$ & $10 \%$ \\
\hline Expectativa de vida* & 40 & 72 & 79 \\
\hline Utilidade & & & \\
0-10 anos & 0,30 & 0,45 & 0,83 \\
11-21 anos & 0,18 & 0,42 & 0,73 \\
$>21$ anos & 0,30 & 0,42 & 0,79 \\
\hline QALY & 10,50 & 30,12 & 61,42 \\
\hline
\end{tabular}

QALY: Quality-Adjusted Life Years. * Dados extraídos de YHEC, 2015 (YHEC, 2015).

Tabela 3. Custos médios atribuídos a cada um dos eventos

\begin{tabular}{lc}
\hline Eventos & Custos (R\$) \\
\hline Defeitos de fechamento do tubo neural* & $17.949,95$ \\
\hline Pré-eclâmpsia & $1.405,12$ \\
\hline Prematuridade & $5.904,16$ \\
\hline Anemia & 155,22 \\
\hline
\end{tabular}

* Considerados custos de interrupção de gestação, nascimento com anencefalia e espinha bífida, na proporção de $80 \%, 6,67 \%$ e 13,33\%, respectivamente (Bhutta \& Khan, 2004).

Tabela 4. Quantidade de eventos relacionados à não utilização de suplementação vitamínica no período de um ano

\begin{tabular}{lc}
\hline Desfechos & Quantidade de eventos \\
\hline \multicolumn{2}{l}{ Defeito no fechamento do tubo neural } \\
\hline Pré-eclâmpsia & 5.352 \\
\hline Cálcio & 144.941 \\
\hline Magnésio & 177.040 \\
\hline Vitamina C & 207.377 \\
\hline Vitamina D & 345.628 \\
\hline Vitamina E & 211.837 \\
\hline Zinco & 158.422 \\
\hline Anemia & 796.283 \\
\hline Parto prematuro & 298.801 \\
\hline QALY (espinha bífida) & 19.801 \\
\hline
\end{tabular}

QALY: Quality-Adjusted Life Years.

* QALY estimado considerando o período de duração da vida (life time).

Já para a anemia em gestantes, a falta de suplementação seria capaz de gerar a ocorrência de 796.283 casos da doença e um custo anual total de $\mathrm{R} \$ 123.599 .056,29$. Considerando o desfecho parto prematuro, seriam observados 298.801 casos por ano em virtude da falta da suplementação com ômega-3, gerando um custo total de R\$ 1.764.170.444,77, o maior gasto entre os desfechos analisados.

Para a ocorrência de pré-eclâmpsia, a falta de estratégias de suplementação com diferentes micronutrientes foi considerada no modelo. Atualmente só existem estudos
Tabela 5. Custos relacionados a não utilização de suplementação vitamínica no período de um ano

\begin{tabular}{lc}
\hline Desfechos & Custos (R\$) \\
\hline Custo total & 2.469 .480 .853 \\
\hline Defeito no fechamento do tubo neural* & 96.062 .083 \\
\hline Pré-eclâmpsia & \\
\hline Cálcio & 203.659 .371 \\
\hline Magnésio & 248.762 .355 \\
\hline Vitamina C & 291.389 .561 \\
\hline Vitamina D & 485.649 .269 \\
\hline Vitamina E & 297.656 .003 \\
\hline Zinco & 222.601 .498 \\
\hline Anemia & 123.599 .056 \\
\hline Parto prematuro & 1.764 .170 .445 \\
\hline
\end{tabular}

* Custos estimados considerando o período de duração da vida (life time).

que estimem a probabilidade de ocorrência de pré-eclâmpsia relacionada à deficiência de cada micronutriente isolado. Portanto, a fim de não sobrepor as estimativas e custos com uma mesma complicação, para o custeio do desfecho pré-eclâmpsia, foi considerado apenas o custo decorrente da deficiência de vitamina $D$, que está associada ao maior número de casos (345.628) de pré-eclâmpsia decorrentes da falta de suplementação.

Considerando a ocorrência de eventos de pré-eclâmpsia e seus respectivos custos gerados pela deficiência de cada substância isolada, a deficiência de cálcio pode gerar a ocorrência de 144.941 casos de pré-eclâmpsia por ano, a de magnésio, de 177.040, a de zinco, de 158.422, e a deficiência das vitaminas C, D e E geram a ocorrência de 207.377, 345.628 e 211.837 casos por ano, respectivamente. Além disso, foi estimado que os custos anuais seriam de $\mathrm{R} \$ 203.659 .371$, $R \$ 248.762 .355, \mathrm{R} \$ 222.601 .498, \mathrm{R} \$ 291.389 .561, \mathrm{R} \$ 485.649 .269$ e R\$297.656.003, associados à deficiência de cálcio, magnésio, zinco e vitaminas C, D e E, respectivamente.

A Tabela 4 apresenta, ainda, a estimativa de QALY para indivíduos nascidos com espinha bífida. Aqueles que não recebem suplementação vitamínica apresentam estimativa de 19.801 QALY.

\section{Discussão}

Este estudo foi conduzido com o objetivo de avaliar o impacto da falta de suplementação com mulvitamínicos, por meio da estimativa do número de casos e dos custos implicados no tratamento de complicações médicas decorrentes da falta de suplementação em mulheres no período reprodutivo e recém-nascidos. Essa estimativa é capaz de gerar relevante informação sobre a necessidade de adoção de condutas para a prática clínica, com base no conhecimento de ambos os aspectos. Análises econômicas de desfechos em saúde 
são fundamentais para auxiliar esse processo decisório, uma vez que ponderam a relação entre os benefícios clínicos de diferentes tecnologias e os custos associados à sua adoção (Secoli et al., 2010).

A deficiência de micronutrientes tem se mostrado como um importante desafio para a saúde pública, especialmente em países em desenvolvimento e subdesenvolvidos (Bhutta et al., 2013). O custo da deficiência de micronutrientes em crianças de 6 a 59 meses foi avaliado no Paquistão, sob a perspectiva da sociedade, e foi estimado um total de $46 \mathrm{mi}-$ Ihões de dólares em gastos médicos e de 3.222 milhões de dólares em perda de produtividade, representando cerca de 1,5\% do produto interno bruto do país (Wieser et al., 2017). Uma avaliação similar foi conduzida nas Filipinas e foi estimado um custo total de 30,3 milhões de dólares com gastos médicos e de 618,2 milhões de dólares com perda de produtividade (Wieser et al., 2013). Não foram localizados pelos autores, até o momento, outros estudos que avaliem o impacto dessa condição em mulheres no período reprodutivo e em recém-nascidos, evidenciando a relevância dos achados apresentados.

Na presente análise, foi estimado que a deficiência de diferentes micronutrientes pode gerar um impacto econômico para o SUS de até R\$ 2,47 bilhões, sendo o parto prematuro e a pré-eclâmpsia responsáveis por cerca de $91 \%$ desses gastos.

Apesar de os dados apresentados terem sido gerados com base em estimativas para cada um dos micronutrientes de interesse, a deficiência do conjunto de substâncias parece ser mais relevante do que aquela observada individualmente. Especialmente para a ocorrência de pré-eclâmpsia, em que, apesar de um maior número de casos e custos associados à deficiência de vitamina D ser observado, a importância do papel de micronutrientes como cálcio, magnésio, vitamina C, vitamina $E$ e zinco é destacada. Dessa forma, os dados ora apresentados demonstram o benefício da suplementação com multivitamínicos para essa população.

A utilização de suplementação vitamínica com diferentes substâncias isoladas para a prevenção dos diferentes desfechos de interesse em mulheres no período reprodutivo e recém-nascidos tem sido amplamente avaliada por diferentes autores e apresentam resultados satisfatórios (De-Regil et al., 2016; Hofmeyr et al., 2018; Makrides et al., 2014; Middleton et al., 2018; Ota et al., 2015; Peña-Rosas et al., 2015; Rumbold et al., 2015a; Rumbold et al., 2015b; Salam et al., 2015). No entanto, de acordo com os resultados apresentados neste estudo, essa população poderia se beneficiar da utilização de múltiplas vitaminas em conjunto. De acordo com uma metanálise previamente publicada, mulheres que receberam suplementação com multivitamínicos durante o período gestacional apresentaram menor risco de terem filhos com baixo peso ao nascer e bebês pequenos para a idade gestacional de maneira significativa, quando comparadas àquelas que fizeram uso isolado de ferro e ácido fólico. Outros desfechos, como prematuridade, aborto espontâneo, mortalidade materna e neonatal, mortalidade perinatal e casos de natimortos, não apresentaram diferenças com significância estatística (Bhutta \& Khan, 2004). Há uma defasagem de estudos que avaliam a relação entre custo e efetividade da suplementação com multivitamínicos, dessa forma, outros estudos ainda precisam ser conduzidos.

Apesar dos relevantes achados apresentados, este estudo apresenta limitações. A estratégia de microcusteio com padrão de uso de recursos definido por um especialista clínico foi utilizada, porém essa opinião pode não refletir a realidade observada em um contexto de mundo real e, assim como em outros estudos econômicos, os dados apresentados são derivados de estimativas. Outra importante limitação está relacionada a uma possível sobreposição de ocorrência dos desfechos, dado que um mesmo indivíduo pode apresentar deficiência de múltiplas vitaminas ao mesmo tempo, o que é observado especialmente nas estimativas de pré-eclâmpsia. Para contornar o efeito dessa sobreposição no custo total, apenas o custo da pré-eclâmpsia associado à deficiência de vitamina $D$ foi incluído no cálculo. Além disso, a análise contabilizou apenas as decorrências da ausência de suplementação com os micronutrientes que apresentavam artigos com probabilidades de ocorrência dos desfechos de interesse em gestantes/recém-nascidos bem-definidos. Adicionalmente só foram localizados dados de QALY para o desfecho espinha bífida.

\section{Conclusão}

Após a condução deste estudo, é possível concluir que a falta de suplementação de micronutrientes é capaz de gerar um custo de até $\mathrm{R} \$ 2,47$ bilhões para o sistema brasileiro de saúde pública (SUS). Dependendo do desfecho observado, a ocorrência de casos relacionados à deficiência de vitaminas pode ser superior a 796 mil por ano. Além disso, esses impactos estão relacionados à deficiência de múltiplas vitaminas, demonstrando um potencial benefício do uso de suplementação com multivitamínicos dessa população.

Dessa forma, é possível concluir que o uso da suplementação com multivitamínicos é uma opção a ser considerada pelos médicos para mulheres nos períodos pré-concepcional, gestacional e de amamentação e para crianças de até 6 meses.

\section{Agradecimentos}

Agradecemos à Sense Company pelo apoio com a redação médica durante o desenvolvimento do esboço deste manuscrito. Esse apoio foi financiado pela Bayer.

\section{Referências bibliográficas}

Agência Nacional de Saúde Suplementar - ANS. Beneficiários de planos privados de saúde, por cobertura assistencial (Brasil - 2009-2019). Available from: https://www.ans.gov.br/perfil-do-setor/dados-gerais. Accessed on: Sept. 272019. 
Aguiar MJ, Campos AS, Aguiar RA, Lana AM, Magalhães RL, Babeto LT. Neural tube defects and associated factors among liveborn and stillborn infants. J Pediatr (Rio J). 2003;79(2):129-34.

Brasil. Ministério da Saúde. Sinasc - Sistema de Informações de Nascidos Vivos [Internet]. Portal da Saúde. [cited 2019 Jun 3]. Available from: http://tabnet.datasus.gov.br/cgi/tabcgi.exe?sinasc/cnv/nvuf.def.

Bhutta ZA, Salam RA, Das JK. Meeting the challenges of micronutrient malnutrition in the developing world. Br Med Bull. 2013;106:7-17.

Bhutta Z, Khan I. Multiple-micronutrient supplementation for women during pregnancy. In: Bhutta Z, editor. Cochrane Database of Systematic Reviews. Chichester, UK: John Wiley \& Sons, Ltd; 2004.

Czeizel AE. Periconceptional folic acid containing multivitamin supplementation. Eur J Obstet Gynecol Reprod Biol. 1998;78(2):151-61

De-Regil LM, Palacios C, Lombardo LK, Peña-Rosas JP. Vitamin D supplementation for women during pregnancy(Review). Cochrane Database Syst Rev. 2016;(1):CD008873.

Desgualdo CM, Riera R, Zucchi P. Cost estimate of hospital stays for premature newborns in a public tertiary hospital in Brazil. Clinics. 2011;66(10):1773-7.

Fu Z, Ma ZZ, Liu GJ, Wang LL, Guo Y. Vitamins supplementation affects the onset of preeclampsia. J Formos Med Assoc. 2018;117(1):6-13.

Fujimori E, Sato APS, Szarfarc SC, da Veiga GV, de Oliveira VA, Colli C, et al. Anemia em gestantes brasileiras antes e após a fortificação das farinhas com ferro. Rev Saúde Pública 2011;45(6):1027-35.

United Nations Children's Fund (Unicef). Investing in the future. A united call to action on vitamin and mineral deficiencies. Global Report 2009. Washington (DC): Unicef; 2009. 52p.

Giordano JC, Parpinelli MA, Cecatti JG, Haddad SM, Costa ML, Surita FG, et al. The burden of eclampsia: Results from a multicenter study on surveillance of severe maternal morbidity in Brazil. PLoS One. 2014;9(5):e97401.

Hanson M, Bardsley A, De-Regil LM, Moore SE, Oken E, Poston L, et al. The International Federation of Gynecology and Obstetrics (FIGO) recommendations on adolescent, preconception, and maternal nutrition: "Think Nutrition First". Int J Gynaecol Obstet. 2015;131 Suppl 4:S213-53.

Hofmeyr G, Lawrie TA, Atallah AN, Torloni MR. Calcium supplementation during pregnancy for preventing hypertensive disorders and related problems (Review). Cochrane Database Syst Rev. 2018;(10):CD001059.

Howson CP, Kennedy ET, Horwitz A, editors. Prevention of Micronutrient Deficiencies: Tools for Policymakers and Public Health Workers. Prevention of Micronutrient Deficiencies. Washington (DC): National Academies Press (US); 1998.

Karakochuk CD, Whitfeld KC, Green TJ, Kraemer K, editors. The biology of the first 1,000 days. New York: CRC Press; 2018. v. 24.

Makrides M, Crosby DD, Bain E, Crowther CA. Magnesium supplementation in pregnancy (Review). Cochrane Database Syst Rev. 2014;(4):CD000937.

Middleton P, Gomersall JC, Gould JF, Shepherd E, Olsen SF, Makrides M. Omega-3 fatty acid addition during pregnancy (Review). Cochrane Database Syst Rev. 2018;(11):CD003402.
Mozetic RM, Silva SDC, Ganen AP. A importância da nutrição nos primeiros mil dias. Rev Eletrônica Acervo Saúde. 2016;8(2):876-84.

Mujica-Coopman MF, Brito A, López de Romaña D, Ríos-Castillo I, Coris H, Olivares M. Prevalence of Anemia in Latin America and the Caribbean. Food Nutr Bull. 2015;36(2 Suppl):S119-28.

Ota E, Mori R, Middleton P, Tobe-Gai R, Mahomed K, Miyazaki C, et al. Zinc supplementation for improving pregnancy and infant outcome (Review). Cochrane Database Syst Rev. 2015;(2):CD000230.

Peña-Rosas J, De-Regil LM, Garcia-Casal MN, Dowswell T. Daily oral iron supplementation during pregnancy (Review). Cochrane database Syst Rev. 2015;(7):CD004736.

Rosenthal J, Casas J, Taren D, Alverson CJ, Flores A, Frias J. Neural tube defects in Latin America and the impact of fortification: a literature review. Public Health Nutr. 2014;17(3):537-50.

Rumbold A, Ota E, Hori H, Miyazaki C, Crowther CA. Vitamin E supplementation in pregnancy (Review). Cochrane Database Syst Rev. 2015;(9):CD004069.

Rumbold A, Ota E, Nagata C, Shahrook S, Crowther CA. Vitamin C supplementation in pregnancy (Review). Cochrane Database Syst Rev. 2015;(9):CD004072.

Salam R, Zuberi NF, Bhutta ZA. Pyridoxine (vitamin B6) supplementation during pregnancy or labour for maternal and neonatal outcomes (Review). Cochrane Database Syst Rev. 2015;(6):CD000179.

Santos LM, Lecca RC, Cortez-Escalante JJ, Sanchez MN, Rodrigues HG. Prevention of neural tube defects by the fortification of flour with folic acid: a population-based retrospective study in Brazil. Bull World Health Organ. 2016;94(1):22-9.

Say L, Chou D, Gemmill A, Tunçalp Ö, Moller AB, Daniels J, et al. Global causes of maternal death: a WHO systematic analysis. Lancet Glob Health. 2014;2(6):e323-33.

Secoli SR, Nita ME, Ono-Nita SK, Nobre M. Avaliação de tecnologia em saúde. II. A análise de custo-efetividade. Arq Gastroenterol. 2010;47(4):329.

Sibai B, Dekker G, Kupferminc M. Pre-eclampsia. Lancet. 2005;365(9461):789-99.

Soma-Pillay P, Nelson-Piercy C, Tolppanen H, Mebazaa A. Physiological Changes in Pregnancy. Cardiovasc J Afr. 2016;27(2):89-94.

Wieser S, Brunner B, Tzogiou C, Plessow R, Zimmermann MB, Farebrother J, et al. Societal Costs of Micronutrient Deficiencies in 6- to 59-month-old Children in Pakistan. Food Nutr Bull. 2017;38(4):485-500.

Wieser S, Plessow R, Eichler K, Malek O, Capanzana MV, Agdeppa I, et al. Burden of micronutrient deficiencies by socio-economic strata in children aged 6 months to 5 years in the Philippines. BMC Public Health. 2013;13:1167.

Yi Y, Lindemann M, Colligs A, Snowball C. Economic burden of neural tube defects and impact of prevention with folic acid: A literature review. Eur J Pediatr. 2011;170(11):1391-400.

York Health Economics Consortium (YHEC). National Institute for Health and Care Excellence: Examining the Cost-Effectiveness of Moving the Healthy Start Vitamin Programme from a Targeted to a Universal Offering: Cost- Effectiveness Systematic Review. York: University of York; 2015. 110p. 